\title{
MicroRNA-664a-3p inhibits the proliferation of ovarian granulosa cells in polycystic ovary syndrome and promotes apoptosis by targeting BCL2A1
}

\author{
Min He, Ganghong Mao, Yungai Xiang, Pengfen Li, Yuanyuan Wu, Dongmei Zhao, Tan Li \\ Department of Reproductive Medicine, The Second Affiliated Hospital of Zhengzhou University, Zhengzhou, China \\ Contributions: (I) Conception and design: M He, G Mao, T Li; (II) Administrative support: Y Xiang, P Li, Y Wu; (III) Provision of study materials \\ or patients: Y Wu, D Zhao; (IV) Collection and assembly of data: All authors; (V) Data analysis and interpretation: M He, G Mao, T Li; (VI) \\ Manuscript writing: All authors; (VII) Final approval of manuscript: All authors. \\ Correspondence to: Li Tan. Department of Reproductive Medicine, The Second Affiliated Hospital of Zhengzhou University, Zhengzhou 450014, \\ China. Email: litan668@126.com.
}

Background: To investigate whether micro ribonucleic acid-664a-3p (miR-664a-3p) targeting BCL2A1 affects the proliferation and apoptosis of ovarian granulosa cells.

Methods: Real-time quantitative polymerase chain reaction (qRT-PCR) was used to detect the expression of miR-664a-3p in granulosa cells, granular tumor cell lines (KGN), and normal ovarian epithelial cell lines (IOSE80) in the polycystic ovary syndrome (PCOS) group and the control group. After overexpressing miR664a-3p or inhibiting its expression in KGN cells, qRT-PCR and Western blotting were used to detect the messenger RNA (mRNA) and protein levels of related genes. At the same time, a cell counting kit-8 (CCK8 ) and flow cytometer were used to detect cell proliferation and apoptosis. The TargetScan website was used to predict the potential binding sites of miR-664a-3p and B-cell lymphoma 2-related protein A1 (BCL2A1), which was further verified by qRT-PCR, Western blotting, and the luciferase reporter gene method.

Results: The expression of miR-664a-3p was significantly decreased in both PCOS tissues and KGN cells (both $\mathrm{P}<0.05$ ), and the overexpression of miR-664a-3p inhibited the proliferation of KGN cells and induced their apoptosis. Moreover, our results confirmed that miR-664a-3p directly targets BCL2A1 $(\mathrm{P}<0.05)$, and the inhibitory effect of miR-664a-3p on KGN cells was reversed by BCL2A1 overexpression (both $\mathrm{P}<0.05$ ). The up-regulation of BCL2A1 promotes cell proliferation and reduces cell apoptosis by the mitogenactivated protein kinase/extracellular signal-regulated kinase (MAPK/ERK) pathway (both $\mathrm{P}<0.05$ ).

Conclusions: The up-regulation of miR-664a-3p inhibits the proliferation of KGN cells and increases apoptosis by down-regulating the expression of BCL2A1 and blocking the MAPK/ERK signaling pathway.

Keywords: Polycystic ovary syndrome (PCOS); ovarian granulosa cells; micro ribonucleic acid-664a-3p (miR664a-3p); B-cell lymphoma 2-related protein A1 (BCL2A1); proliferation; apoptosis

Submitted Mar 10, 2021. Accepted for publication May 06, 2021.

doi: $10.21037 /$ atm-21-1614

View this article at: http://dx.doi.org/10.21037/atm-21-1614

\section{Introduction}

Polycystic ovary syndrome (PCOS) is a common reproductive endocrine disease, with a prevalence rate of $4-18 \%$ in women of childbearing age (1). PCOS is characterized by hyperandrogenemia, insulin resistance, rare or anovulatory ovulation, and polycystic ovarian changes. It is also accompanied by clinical symptoms of elevated androgen such as hirsutism and acne (2). Previous studies have shown that PCOS is related to infertility. Furthermore, it will increase the risk of metabolic disorders, promote the occurrence and development of diabetes, obesity, dyslipidemia, and cardiovascular diseases (3), which 
Table 1 Primer sequence of target gene

\begin{tabular}{lll}
\hline Target gene & Forward primer (5'-3') & Reverse primer (5'-3') \\
\hline miR-664a-3p & ACACTCCAGCTGGGTATTCATTTATCCCCAGCC & GCGAGCACAGAATTAATACGAC \\
U6 & GCTTCGGCAGCACATATACTAAAAT & CGCTTCACGAATTTGCGTGTCAT \\
BCL2A1 & TACAGGCTGGCTCAGGACTAT & CGCAACATTTGTAGCACTCTG \\
$\beta$-actin & TTCGAGCAAGAGATGGCCA & CGTACAGGTCTTGGGGAT \\
\hline
\end{tabular}

seriously affects the physical and mental health of women. PCOS is a primary follicular disease. The etiology is not yet clear, and most of the current research focuses on genetics, environment, chemotherapeutic drugs, etc. (4). In recent years, studies have found that there are differences in the expression of miRNAs between PCOS patients and healthy women, suggesting that miRNAs may play an important role in the occurrence and development of PCOS (4). The proliferation and apoptosis of granulosa cells are the root causes of abnormal follicular function (5). Therefore, studying the proliferation and apoptosis mechanisms of ovarian granulosa cells is crucial to the clinical diagnosis and treatment of PCOS.

Micro ribonucleic acids (miRNAs) are small non-coding RNAs that are mainly involved in the post-transcriptional regulation of organisms. They bind to the 3'-UTR of target messenger RNA (mRNA) and negatively regulate gene expression at the post-transcriptional level, which may eventually lead to cell function degradation or related gene translation inhibition (6). Studies have shown that a variety of microRNAs are involved in the regulation of ovarian granulosa cell proliferation and apoptosis (7), and they also play an important regulatory role in ovarian reproduction and endocrine functions. Abnormal expression or dysfunction of ovarian microRNA may affect follicular development and atresia, as well as hormone synthesis and metabolism, and ultimately lead to the occurrence and development of ovarian diseases such as PCOS (7). Micro (miR)-664a-3p is located in the intron of RAB3GAP2 and is reported to be abnormally expressed in various malignant tumors, including cervical cancer, osteosarcoma, breast cancer, and T-cell acute lymphoblastic leukemia $(8,9)$. However, the exact role and mechanism of MiR-664a-3p in PCOS have not yet been elucidated.

B-cell lymphoma 2-related protein A1 (BCL2A1) is a member of the BCL-2 family of anti-apoptotic proteins, and is related to resistance to chemotherapy and targeted drugs (10). Studies have found that BCL2A1 plays the role of specific oncogenes by blocking cell death $(11,12)$. According to relevant domestic and foreign literature, rare studies have explored the correlation between PCOS and BCL2A1 or miR-664a-3p. Therefore, this study aims to investigate the correlation between PCOS, miR-664a-3p, and BCL2A1, as well as observe the effects of miR-664a-3p and BCL2A1 on ovarian granulosa cells. By exploring the mechanism of miR-664a-3p and BCL2A1 in the occurrence and development of PCOS, the results of this study will help provide new ideas for the clinical diagnosis and targeted therapy of PCOS. We present the following article in accordance with the MDAR reporting checklist (available at http://dx.doi.org/10.21037/atm-21-1614).

\section{Methods}

\section{Clinical samples}

One hundred and fifty subjects (80 PCOS patients and 70 control patients) who received intracytoplasmic sperm injection or in vitro fertilization in our hospital from April 2018 to April 2020 were included in the study. PCOS was diagnosed according to the revised Rotterdam Consensus, and women with normal menstruation and ovarian function were used as controls (13). The specific clinical and endocrine information of PCOS and control patients are shown in Table 1 . Tissue was collected from each participant and stored at $-80^{\circ} \mathrm{C}$ for subsequent research. All procedures performed in this study involving human participants were in accordance with the Declaration of Helsinki (as revised in 2013). The study was approved by the Ethics Committee of The Second Affiliated Hospital of Zhengzhou University (No.: 2017663) and informed consent was taken from all the patients.

\section{Cell culturing}

IOSE80 cells (Shanghai Fuheng Cell Center, Shanghai, 
China) were cultured in Dulbecco's Modified Eagle Medium (DMEM; Gibco, USA). Human ovarian granulosa cell tumor cells (KGN cells) (Shanghai Fuheng Biotechnology Co., Ltd., China) were cultured in DMEM/F-12 (Gibco, USA) supplemented with $10 \%$ fetal bovine serum (Gibco, USA) and $1 \%$ penicillin-streptomycin (Gibco, USA), and incubated in a $5 \% \mathrm{CO}_{2}$ incubator at $37^{\circ} \mathrm{C}$. At passage, the cells were first digested with $0.25 \%$ trypsin (containing ethylene diamine tetraacetic acid, Gibco, USA). Next, the cell suspension was evenly distributed into $25 \mathrm{~cm}^{2}$ cell culture flasks, and $6 \mathrm{~mL}$ of culture medium was added to each flask.

\section{Cell transfection}

The well-grown $2 \times 10^{5}$ cells were seeded on a six-well plate. When the cell fusion rate reached $80 \%$, the instructions of Lipofectamine ${ }^{\mathrm{TM}} 2000$ (Invitrogen, USA) were followed to perform miR-664a-3p mimic (mimic), miR-664a-3p inhibitor (inhibitor), negative control (negative control, NC), BCL2A1 overexpression plasmid (pc-BCL2A1), and empty plasmid (vector) cell transfection. The above vector plasmids were all constructed by Sangon Biotech (Shanghai, China) Co., Ltd. After 6-8 h of transfection, the medium was changed. After $48 \mathrm{~h}$, the transfection become stable and follow-up research was conducted.

\section{The detection of cell proliferation using the cell counting kit-8 (CCK-8) method}

After transfection, cells in the log phase were trypsinized and resuspended in a complete medium. $100 \mu \mathrm{L}\left(10^{4}\right.$ cells $)$ of cell suspension was added to each well of a 96-well culture plate. After culturing for $0,24,48,72$, and $96 \mathrm{~h}$, $10 \mu \mathrm{L}$ of CCK-8 reagent (Dojindo, Japan) was added to each well, which were then placed back into the incubator and incubated for $2 \mathrm{~h}$. Subsequently, a spectrophotometer (Huawei Delang Instruments Co., Ltd., Wuxi, China) was used to detect the absorbance at $450 \mathrm{~nm}$.

\section{Cell cycle detection using flow cytometry}

After $48 \mathrm{~h}$ of transfection, KGN cells were collected, washed with pre-cooled phosphate buffer saline (PBS), and trypsinized. Next, the cell pellet was collected by centrifugation at $1,000 \mathrm{~g}$ for $5 \mathrm{~min}$ at room temperature and the supernatant was discarded. After washing twice with
PBS, the PBS was discarded and $70 \%$ ethanol was added for fixation, which was subsequently placed in a refrigerator at $-20{ }^{\circ} \mathrm{C}$ for $48 \mathrm{~h}$. After routine propidium iodide (PI) staining (Invitrogen, USA), the deoxyribonucleic acid (DNA) content of the cells was measured by flow cytometry, and the percentage of each phase of the cell cycle was then analyzed using Flowjo software (Treestar, Inc., USA).

\section{The detection of apoptosis by flow cytometry}

The Annexin V-FITC/PI Apoptosis Detection Kit (Invitrogen, USA) was used to detect cell apoptosis. The collection and washing of the cells was performed using the same procedure as that used for cell cycle detection. Next, the binding buffer containing Annexin V-FITC/PI was added to resuspend the cells, which were then incubated at room temperature and protected from light for $15 \mathrm{~min}$. Thereafter, the flow cytometer (CytoFlex S, Beckman Coulter, CA, USA) was used for detection immediately, and the apoptosis rate was analyzed using Flowjo software.

\section{Detection of cell protein expression using Western blotting}

Upon completion of the treatment, the cell culture medium was discarded, pre-cooled PBS was added to wash the cells twice, and then $200 \mu \mathrm{L}$ of protein lysate was added (containing $2 \mu \mathrm{L}$ of protease inhibitor and $2 \mu \mathrm{L}$ of phosphatase inhibitor, both purchased from Shanghai Sheng Industrial Biology Co., Ltd.). After ice cracking for 30 minutes, $\beta$-mercaptoethanol-containing protein buffer was added (Shanghai Shenggong Biological Co., Ltd., China) and mixed well, boiled for 15 minutes, and stored at $-20{ }^{\circ} \mathrm{C}$ for later use. The extracted protein samples were separated with $10 \%$ polyacrylamide gel and transferred to a polyvinylidene fluoride (PVDF) membrane (Millipore, USA). After blocking the membrane with $5 \%$ bovine serum albumin (Shanghai Shenggong Biological Co., Ltd., China) for $2 \mathrm{~h}$ at room temperature, it was incubated with primary antibody overnight. It was subsequently incubated with the corresponding secondary antibody for $1 \mathrm{~h}$ the next day. Lastly, the enhanced chemiluminescence method (ECL; Shanghai Shenggong Biological Co., Ltd., China) was used for exposure and development. The primary antibodies used, including CDK2 (1:1,000, ab32147), CCNB1 (1:1,000, ab181593), BCL2A1 (1:1,000, ab45413), BCL2 (1:1,000, ab182858), Bax (1:1,000, ab32503), and $\beta$-actin (1:2,000, ab8226), were purchased from Abcam, USA. 
Table 2 Clinical characteristics of polycystic ovary syndrome (PCOS) patients and healthy controls

\begin{tabular}{|c|c|c|c|}
\hline Variables & Control group $(n=70)$ & $\operatorname{PCOS}(n=80)$ & $P$ value \\
\hline $\mathrm{BMI}\left(\mathrm{kg} / \mathrm{m}^{2}\right)$ & $20.7 \pm 2.4$ & $23.3 \pm 3.1$ & 0.014 \\
\hline Follicle stimulating hormone (mIU/mL) & $6.5 \pm 2.1$ & $5.4 \pm 2.7$ & 0.034 \\
\hline Luteinizing hormone (mlU/mL) & $6.7 \pm 3.4$ & $9.4 \pm 4.5$ & 0.031 \\
\hline Estrogen $(\mathrm{pg} / \mathrm{mL})$ & $31.1 \pm 2.7$ & $79.4 \pm 7.5$ & 0.011 \\
\hline Blood sugar (mmol/mL) & $4.1 \pm 1.6$ & $1.3 \pm 0.3$ & 0.004 \\
\hline Testosterone (nmol/mL) & $1.3 \pm 0.4$ & $2.3 \pm 0.4$ & 0.015 \\
\hline Hyperandrogenemia, n (\%) & $16(22.9)$ & $64(80.0)$ & 0.001 \\
\hline Hyperinsulinemia, n (\%) & $4(5.7)$ & $33(41.3)$ & 0.005 \\
\hline Number of oocytes & $18.3 \pm 2.1$ & $8.3 \pm 2.3$ & 0.001 \\
\hline Number of high-quality embryos & $11.3 \pm 8.1$ & $3.3 \pm 1.8$ & 0.002 \\
\hline
\end{tabular}

\section{Target verification by luciferase reporter gene experiment}

The TargetScan website was used to predict the binding fragment of miR-664a-3p and BCL2A1. The amplified BCL2A1-3'-UTR sequence was inserted into the microRNA reporter luciferase plasmid to construct the BCL2A1-3'-UTR luciferase reporter (wild type, wt) wild type vector BCL2A1-Wt and mutant (mutant type, mut) vector BCL2A1-Mut. The luciferase reporter vector and mutant vector were co-transfected with microRNAnegative control (NC) or microRNA-664a-3p into HEK293T cells, and the luciferase activity was detected in strict accordance with the instructions of the dual-luciferase reporter kit (Promega, USA).

\section{$R N A$ extraction and real-time fuorescent quantitative PCR (qRT-PCR) detection}

Total RNA was extracted with TRIzol (Invitrogen, USA) and reverse transcribed into complementary DNA (cDNA) (Takara, Japan). Next, Power-SYBR-Green (Takara, Japan) was used for fluorescence quantitative detection. $\beta$-actin was used as an internal reference gene. TaqMan Human MiRNA Detection Kit (Invitrogen, Japan) was used for the amplification and detection of miR-664a-3p, and U6 was used as an internal control. The $2^{-\Delta \Delta C t}$ method was used to calculate the relative gene expression level.

\section{Statistical analysis}

All experiments in this study were independently repeated three times. SPSS 20.0 statistical analysis software (IBM, USA) was used for analysis. The student's $t$-test was used for comparison between the two groups, and $\mathrm{P}<0.05$ was considered to indicate statistical significance.

\section{Results}

\section{Clinical and endocrine indicators of PCOS patients and bealthy controls}

The clinical and endocrine indicators of PCOS patients and the control group are shown in Table 2. Compared with the control group, the levels of body mass index (BMI), prolactin, luteinizing hormone, estrogen, androgen, testosterone, and insulin in PCOS patients were significantly increased, and follicle-stimulating hormone was markedly decreased $(\mathrm{P}<0.05)$. There was no significant 

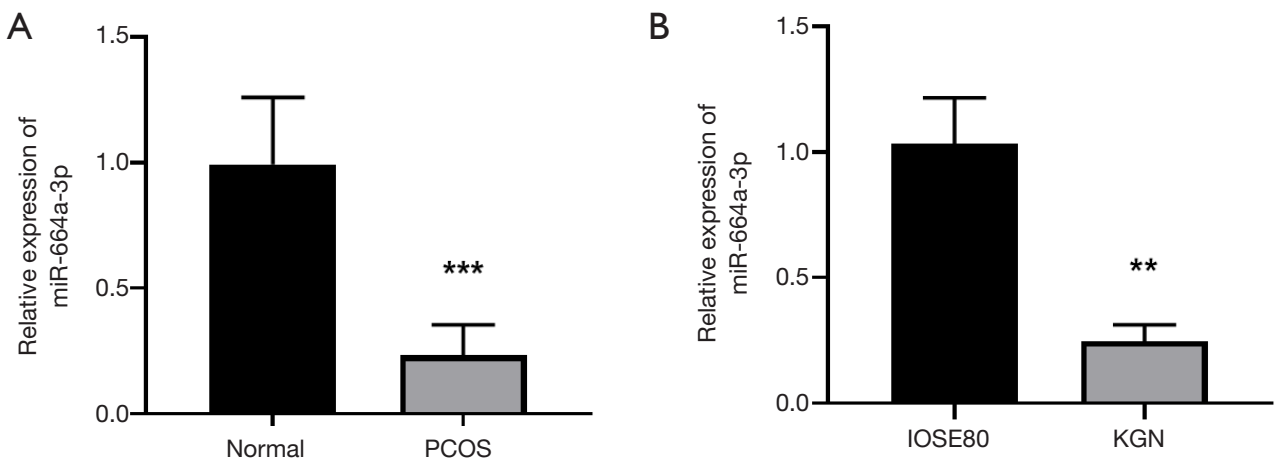

Figure 1 MiR-664a-3p was low in PCOS. (A) The expression of miR-664a-3p in granulosa cells of PCOS patients and healthy controls; (B) the expression of miR-664a-3p in KGN and IOSE80 cells. Compared with the healthy control (normal)/IOSE80 group, ** $\mathrm{P}<0.01$, ${ }^{* * *} \mathrm{P}<0.001$.

difference in age between the two groups $(\mathrm{P}>0.05)$.

\section{The expression of miR-664a-3p in granulosa cells and KGN cells of PCOS patients}

To determine whether miR-664a-3p is related to PCOS, qRT-PCR was used to detect the expression of miR-664a$3 p$ in the granulosa cells (as well as KGN and IOSE80 cells) of 80 PCOS patients and 70 healthy controls. As shown in Figure $1 A$ and $1 B$, the level of miR-664a-3p in the granulosa cells in PCOS patients was significantly lower than that in the control group $(\mathrm{P}<0.05)$. Also, further analysis of the interaction between miR-664a-3p and PCOS showed that the expression of miR-664a-3p in KGN cells was also significantly decreased $(\mathrm{P}<0.05$, Figure $1 B)$.

\section{miR-664a-3p inbibits granulosa cell proliferation}

To verify the hypothesis that miR-664a-3p may be involved in granular cell proliferation, we transiently transfected KGN cells with a miR-664a-3p mimic, inhibitor, and NC. Figure $2 A$ shows the verification of miR-664a-3p expression using a mimic and inhibitor. As shown in Figure 2B, CCK8 detection found that the overexpression of miR-664a$3 p$ inhibited the proliferation of KGN cells in a timedependent manner (all $\mathrm{P}<0.05$ ), while interference with its expression had the opposite effect $($ all $\mathrm{P}<0.05)$. The cell cycle results (Figure 2C) that the overexpression of miR$664 a-3 p$ increased the number of cells in G0/G1 phase and decreased the number of cells in the $\mathrm{S}$ phase $(\mathrm{P}<0.05)$, indicating that the cell cycle was inhibited. Also, compared with the NC group, the expression of cyclin-related proteins CDK2 and CCNB1 in cells transfected with miR664a-3p was significantly downregulated (both $\mathrm{P}<0.05$, Figure $2 D$ and $E$ ). The above results suggest that the expression level of microRNA-664a-3p is related to the occurrence of PCOS.

\section{miR-664a-3p induces apoptosis of granulosa cells}

To further investigate the function of miR-664a-3p in granulosa cells, this study used flow cytometry to analyze the apoptosis of KGN cells after transfection with a miR664a-3p mimic or inhibitor. Moreover, the expression changes of apoptosis-related proteins, BCL2 and Bax, were detected by Western blot. Figure $3 A$ show that miR664a-3p significantly increased the apoptosis rate of KGN cells $(\mathrm{P}<0.05)$, while the miR-664a-3p inhibitor reduced KGN cell apoptosis $(\mathrm{P}<0.05)$. Figure $3 B$ shows that the overexpression of miR-664a-3p markedly reduced BCL2 and increased the expression of the Bax protein (all $\mathrm{P}<0.05$ ). However, the opposite effect was observed when the expression of miR-664a-3p decreased (all $\mathrm{P}<0.05$ ).

\section{$B C L 2 A 1$ is the direct target of miRNA-664a-3p}

To explore the mechanism of miR-664a-3p in granulosa cells, we used the TargetScan website to predict the possible target of the gene. As shown in Figure 4A, there are binding sites between miRNA-664a-3p and BCL2A1. To verify the prediction of TargetScan, a luciferase reporter vector containing the predicted binding site of 


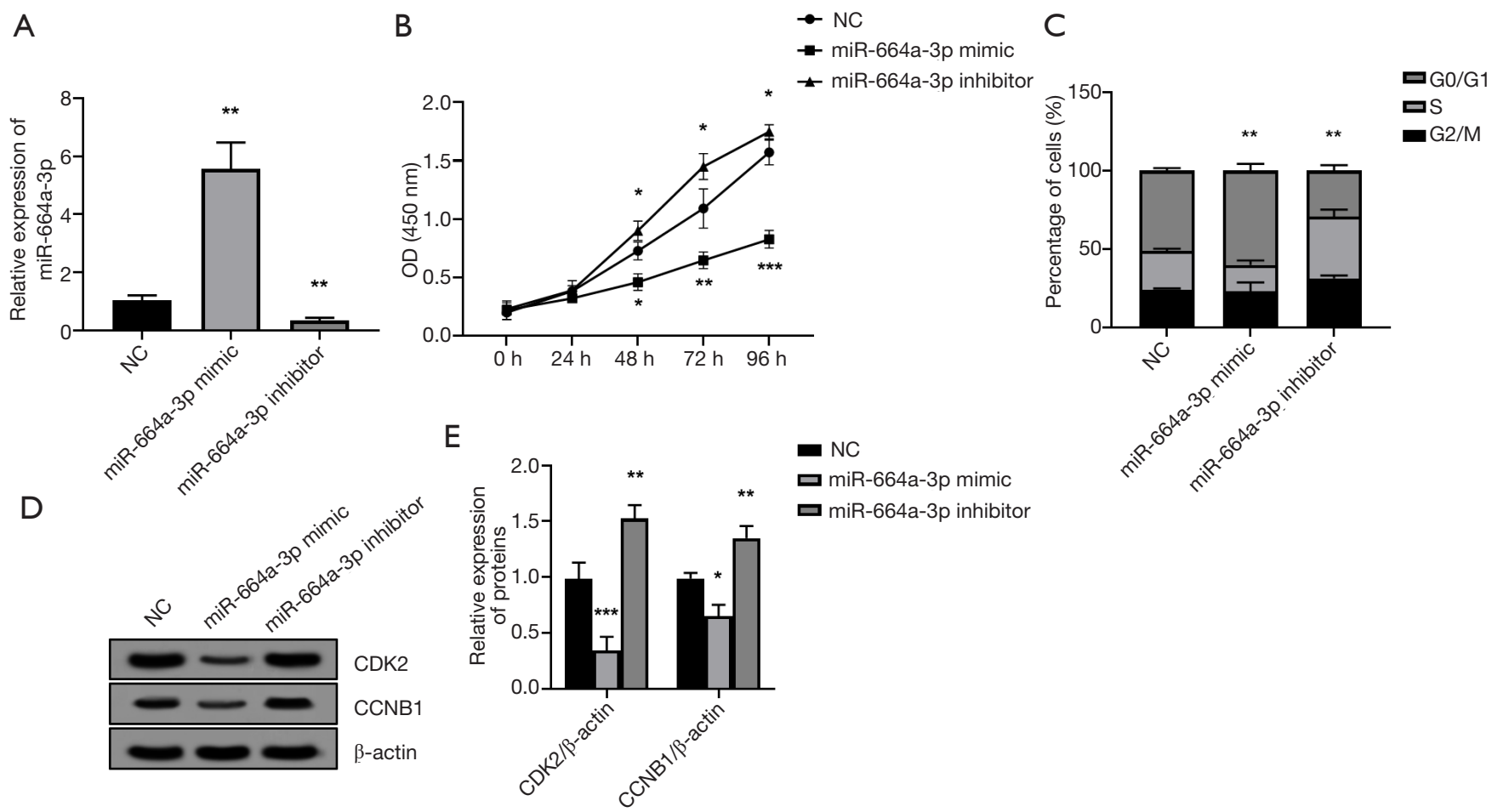

Figure 2 The effect of miR-664a-3p on the proliferation of granulosa cells. (A) The transfection efficiency of the miRNA-664a-3p mimic in KGN cells. (B) The effect of miRNA-664a-3p on the proliferation of KGN cells. (C) The effect of miRNA-664a-3p on the cell cycle of KGN. (D,E) The effect of miRNA-664a-3p on the expression of CDK2 and CCNB1 in KGN cells. Compared with the NC group, ${ }^{*} \mathrm{P}<0.05,{ }^{* *} \mathrm{P}<0.01,{ }^{* *} \mathrm{P}<0.001$.

BCL2A1 3'UTR was constructed in this study. As shown in Figure 4B, after co-transfection with BCL2A1-Wt 3'UTR, the overexpression of miR-664a-3p resulted in a significant decrease in luciferase activity $(\mathrm{P}<0.05)$. Meanwhile, after cotransfection with BCL2A1-Mut 3'UTR, no notable change was observed $(\mathrm{P}>0.05)$. Further exploring the regulatory effect of miR-664a-3p on BCL2A1, this study found that the mRNA and protein expression of BCL2A1 was significantly reduced when KGN cells overexpressed miR664a-3p (both $\mathrm{P}<0.05$, Figure $4 C, D, E$ ).

\section{Verification of BCL2A1 reversing the effect of miR-664a- $3 p$ on KGN cell proliferation and apoptosis}

As shown in Figure $5 A, B, C, D, F$, the overexpression of BCL2A1 reversed the inhibition of miR-664a-3p overexpression on cell proliferation and the cell cycle (all $\mathrm{P}<0.05$ ), and also reversed the effect of miR-664a-3p on the induction of apoptosis (all $\mathrm{P}<0.05)$.

\section{The effect of miR-664a-3p inbibition on the mitogen- activated protein kinase/extracellular signal-regulated kinase (MAPK/ERK) signaling patbway}

Western blot analysis showed that miR-664a-3p inhibited the phosphorylation of MAPK, ERK, and c-Jun N-terminal kinase (JNK) proteins (all $\mathrm{P}<0.05$ ), while the overexpression of BCL2A1 reversed this effect (all $\mathrm{P}<0.05)$ (Figure 6).

\section{Discussion}

PCOS is a complex and multifactorial endocrine disease that affects about $10 \%$ of women of childbearing age (14). It is related to multiple small antral follicles in the ovary, which cannot develop into larger dominant follicles. The ovarian granulosa cells of PCOS are abnormal. Studies have observed an increase in the number of follicles and GC proliferation in mouse PCOS models (15). Furthermore, an increased proliferation of granulosa cells in smaller follicles has also been observed in the ovaries of PCOS women 


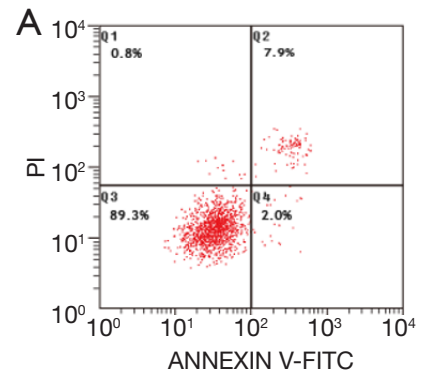

NC

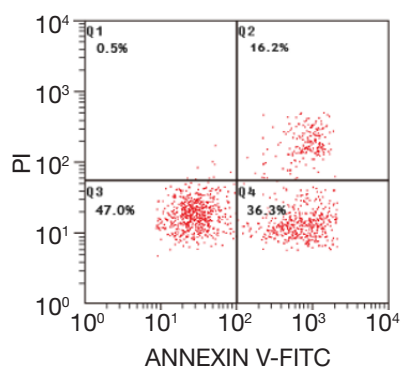

miR-664a-3p mimic

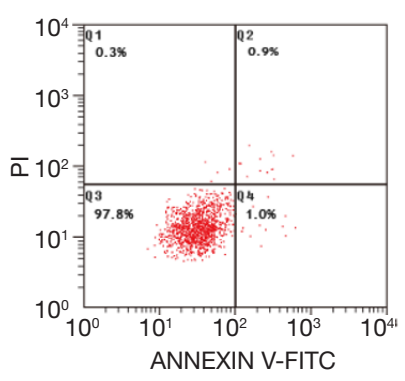

miR-664a-3p inhibitor

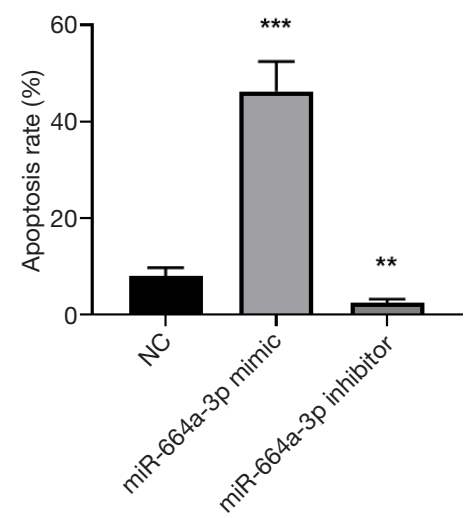

B
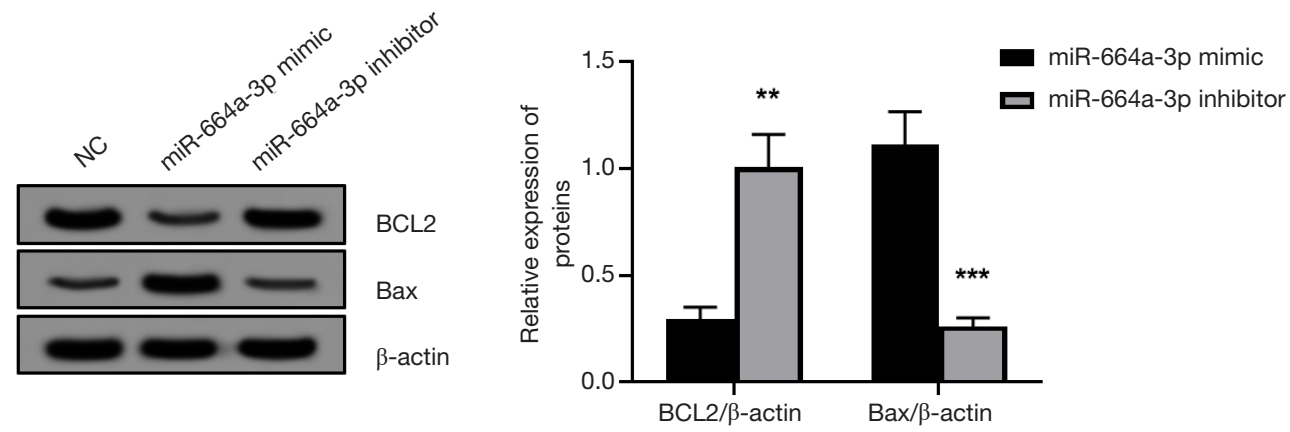

Figure 3 The effect of miR-664a-3p on the apoptosis of granulosa cells. (A) Flow cytometry was used to detect the effect of miRNA-664a3 p on KGN cell apoptosis. (B) Western blotting was used to determine the effect of miRNA-664a-3p on the regulation of apoptosis-related proteins (BCL2 and Bax). Compared with NC group, ${ }^{* *} \mathrm{P}<0.01,{ }^{* * *} \mathrm{P}<0.001$.

$(16,17)$. Therefore, abnormal ovarian GC proliferation may play a role in the pathogenesis of PCOS. However, its internal mechanism is remains unclear. As a group of important post-transcriptional regulators, miRNAs play an important role in granular cells under physiological and pathological conditions. Many miRNAs are expressed in GC and directly regulate the normal development and function of follicles by targeting specific molecules and regulating various signaling pathways (18), including atresia, ovulation, and ovarian steroid production. Moreover, studies have confirmed that miRNAs can play a role in PCOS by affecting granulosa cells (19).

Previous reports indicate that $\mathrm{miR}-664 \mathrm{a}-3 \mathrm{p}$ has different functions in a variety of diseases. Yoneda et al. used microarray technology and RT-PCR technology to analyze the serum miRNA profiles in samples from chronic periodontitis patients and non-chronic periodontitis patients, and found that the expression of miR-664a-3p in patients with periodontitis was higher than that in the control group. MiR-664a-3p is expected to become a serum biomarker for chronic periodontitis (20). Also, Modak et al. found that the expression of miR-664a-3p in patients with cardiogenic stroke is abnormal (21). In addition, abnormal expression of miR-664a-3p has also been found in a variety of malignant tumors such as gastric cancer, breast cancer, cervical cancer, osteosarcoma, and chronic respiratory diseases $(22,23)$. This study first detected the expression of miR-664a-3p in granulosa cells in PCOS patients and healthy controls, and found that its expression was decreased. Subsequently, by overexpressing or interfering with miR-664a-3p in granulosa cells, it was found that the miR-664a-3p has the effect of inhibiting granulosa cell proliferation and promoting apoptosis.

BCL2A1 is one of the least studied members of the BCL2 family. According to its anti-apoptotic activity, BCL2A1 is highly upregulated in several hematopoietic malignancies (24). Recent studies have found that the decreased expression of BCL2A1 in most malignant tumors can restore the tumor's sensitivity to chemotherapy drugs, providing a clear basis for cancer targeting BCL2A1 


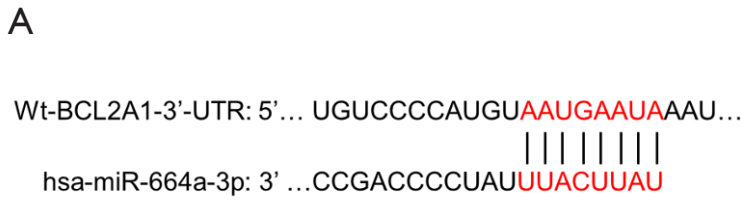

Mut-BCL2A1-3'-UTR: 5'...UGUCCCCAUGUAUCGAUGUAAU...
B

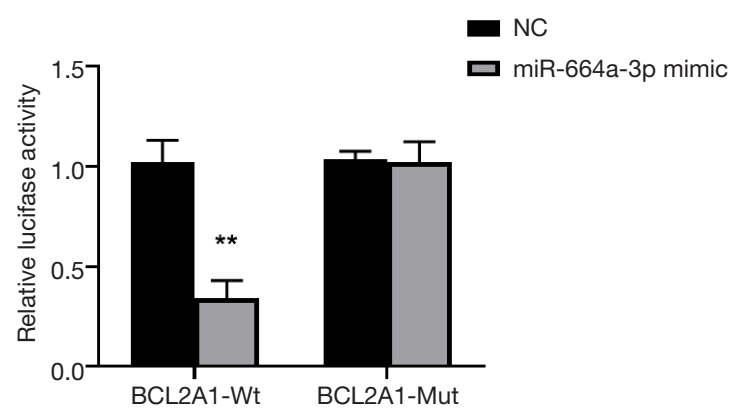

E
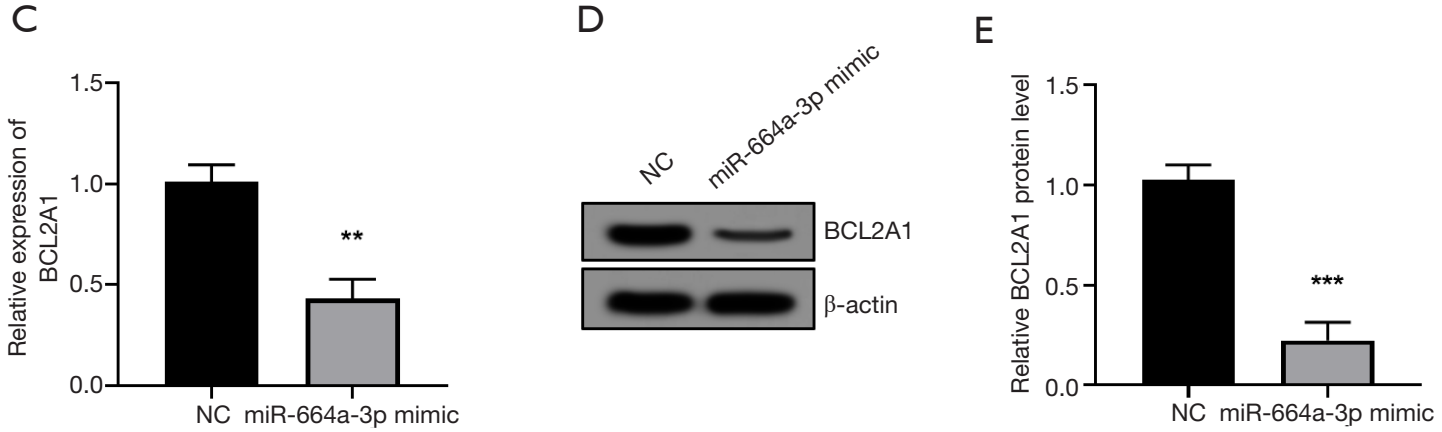

Figure 4 BCL2A1 is a potential target gene verification of miRNA-664a-3p. (A) The potential binding site of miR-664a-3p and BCL2A1. (B) Luciferase reporter gene was used to verify the targeting relationship between the two. (C,D,E) Expression of BCL2A1 when KGN cells overexpressed miR-664a-3p. Compared with the NC group, ${ }^{* *} \mathrm{P}<0.01,{ }^{* * *} \mathrm{P}<0.001$.

$(25,26)$. However, there is no related literature report on BCL2A1 and PCOS. This study first predicted the possible target genes of miR-664a-3p using the biological information website (TargetScan), and BCL2A1 attracted our attention due to its anti-apoptotic activity and potential binding sites. The luciferase reporter gene experiment confirmed that BCL2A1 is indeed the target gene of miR-664a-3p. Subsequently, the co-transfection of the BCL2A1 overexpression plasmid and miR-664a-3p mimic in granulosa cells proved that BCL2A1 could reverse the inhibitory effect of miR-664a-3p on KGN cell proliferation and promote apoptosis. Furthermore, it is known that BCL2A1 has an activating effect on the MAPK signaling pathway (27), and thus, this study was used for verification. Studies have demonstrated that the MAPK pathway of KGN cells overexpressing miR-664a-3p is significantly inhibited, and this inhibition can be reversed when the cells overexpress BCL2A1 at the same time. It is suggested that miR-664a-3p plays a role in KGN cells by inhibiting BCL2A1/MAPK signaling.

This study is the first to use granulosa cells in an in vitro study to confirm that miR-664a-3p can inhibit the proliferation of KGN cells and induce apoptosis. The specific mechanism may be that miR-664a-3p acts by targeting BCL2A1 to regulate the activation state of the MAPK/ERK signaling pathway. These findings provide a theoretical basis for the clinical application of miR-664a$3 p$ in PCOS monitoring and treatment. However, this study also has limitations. For example, this study only used granule cell KGN to confirm the effect of miR-664a-3p in vitro, which was limited to the limitations of basic research. Further in-depth research is still needed to explore the role and mechanism of miR-664a-3p in PCOS, in order to provide new ideas for the clinical treatment of PCOS as soon as possible.

\section{Conclusions}

MiR-664a-3p deactivates the MAPK/ERK signaling pathway by targeting BCL2A1, thereby inhibiting the proliferation of KGN cells and inducing apoptosis. This study provides a possible target for the clinical treatment of PCOS. 


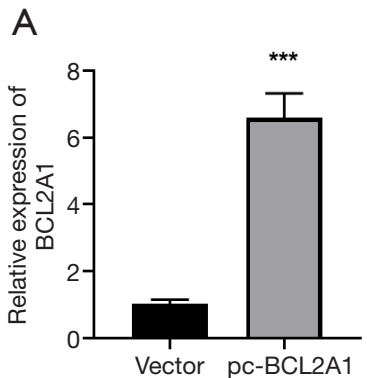

B

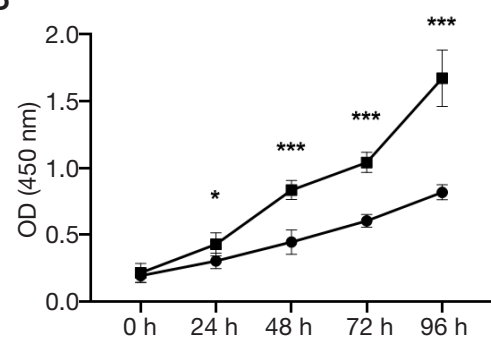

C

$\rightarrow-m i R-664 a-3 p$ mimic + vector

$\rightarrow$ miR-664a-3p mimic + pc-BCL2A

口G0/G1

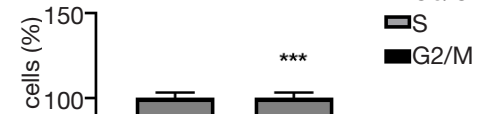

D

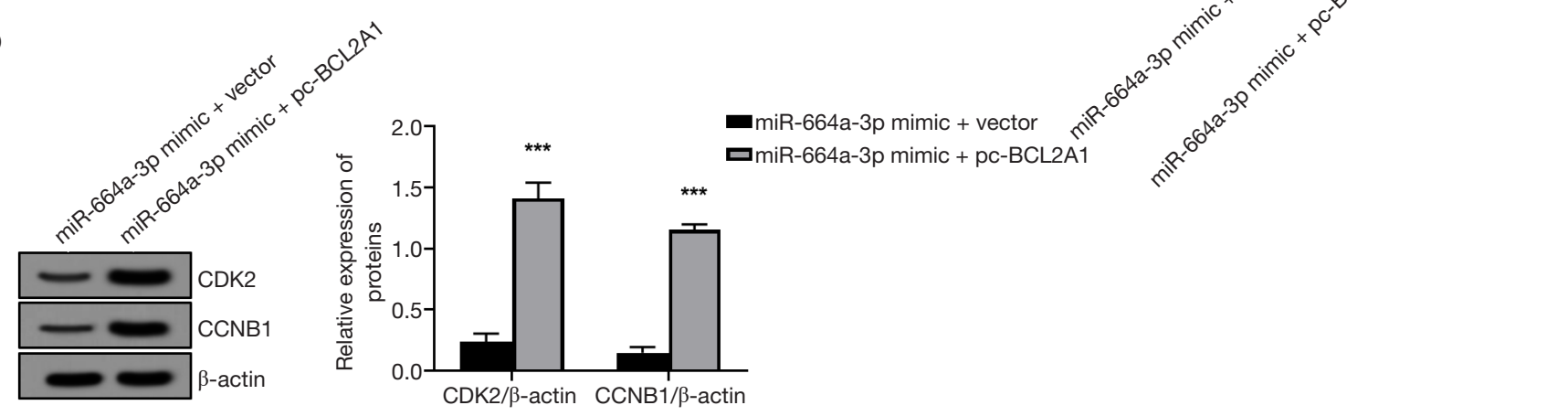

$\mathrm{E}$
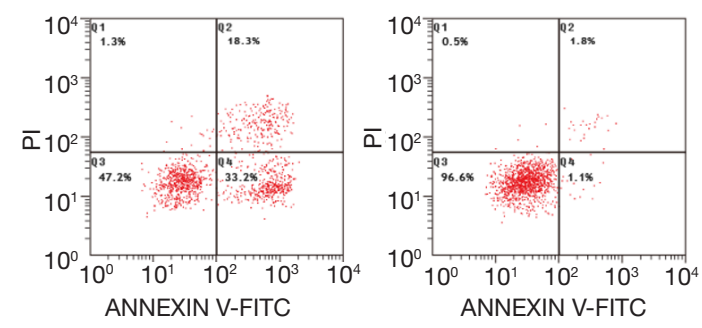

miR-664a-3p mimic + vector

miR-664a-3p mimic + pc-BCL2A1

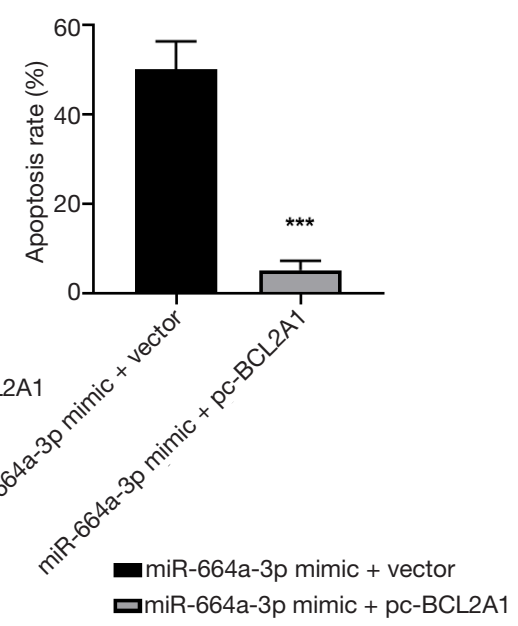

$\mathrm{F}$

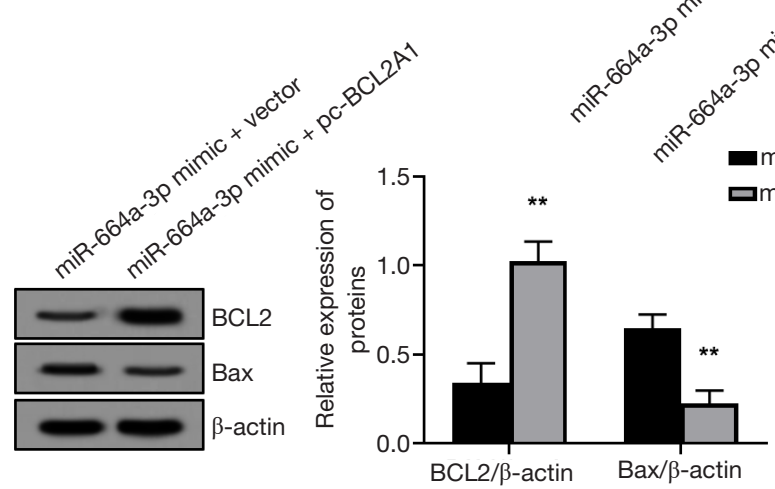

Figure 5 Overexpression of BCL2A1 can reverse the effect of miR-664a-3p on granular cell function. (A) Verification of the efficiency of overexpression of the BCL2A1 plasmid; (B) overexpression of BCL2A1 reversed the effect of miRNA-664a-3p on the proliferation of KGN cells; (C) overexpression of BCL2A1 reversed the effect of miRNA-664a-3p on the cell cycle of KGN; (D) overexpression of BCL2A1 reversed the effect of miRNA-664a-3p on KGN cells expressing cell cycle-related proteins CDK2 and CCNB1; (E) overexpression of BCL2A1 reversed the effect of miRNA-664a-3p on KGN cell apoptosis; (F) overexpression BCL2A1 reversed the effect of miRNA-664a$3 \mathrm{p}$ on the expression of apoptosis-related proteins, BCL2 and Bax, in KGN cells. Compared with the vector group, ${ }^{*} \mathrm{P}<0.05,{ }^{* *} \mathrm{P}<0.01$, ${ }^{* * *} \mathrm{P}<0.001$. N.S.: $\mathrm{P}>0.05$. N.S, no significant. 


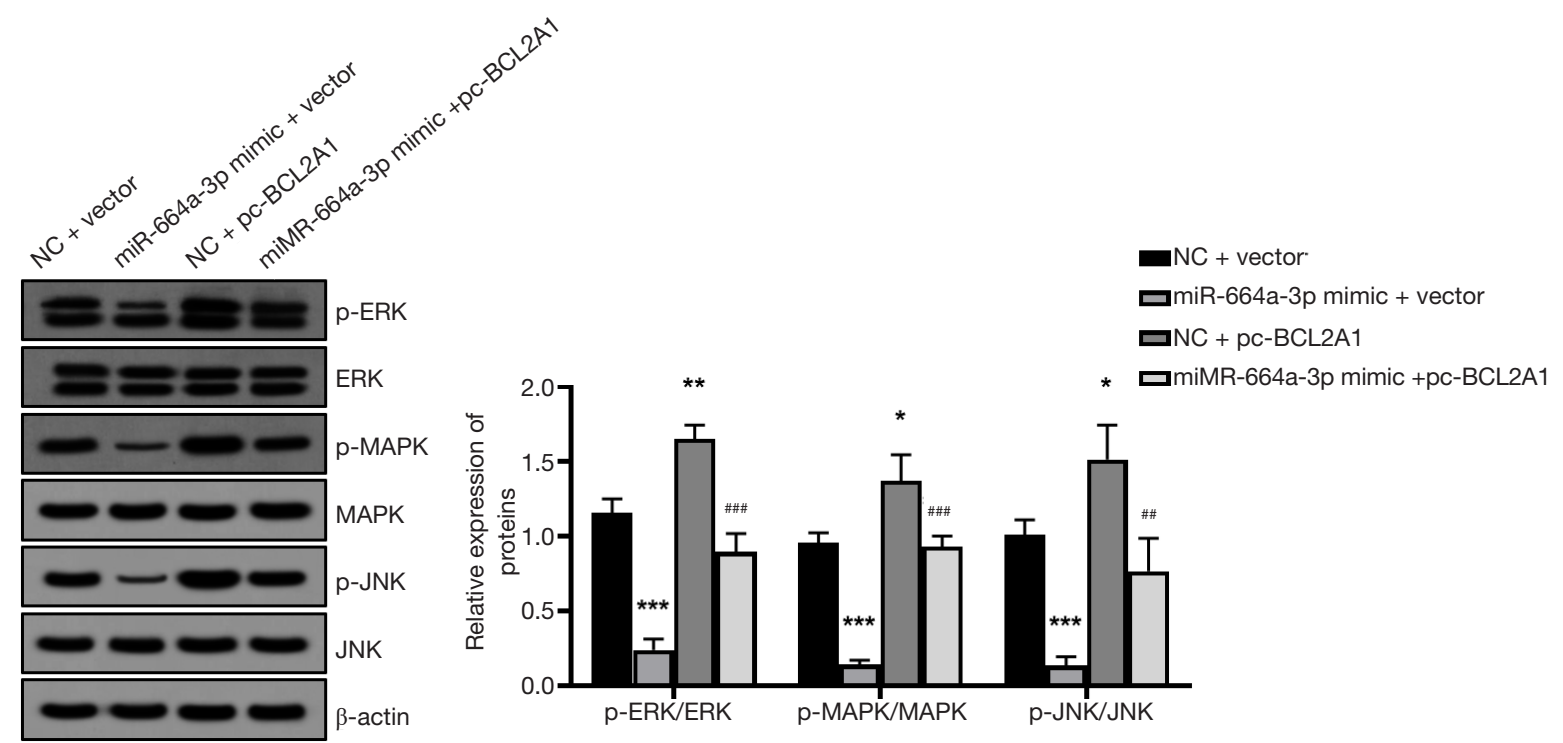

Figure 6 The inhibitory effect of miR-664a-3p on the MAPK/ERK signaling pathway. Compared with $\mathrm{NC}+\mathrm{vector}$ group, ${ }^{*} \mathrm{P}<0.05$, ${ }^{* *} \mathrm{P}<0.01,{ }^{* * *} \mathrm{P}<0.001$; compared with miR-664a-3p + vector group, ${ }^{* \#} \mathrm{P}<0.01,{ }^{\# \#} \mathrm{P}<0.001$.

\section{Acknowledgments}

Funding: None.

\section{Footnote}

Reporting Checklist: The authors have completed the MDAR reporting checklist. Available at http://dx.doi.org/10.21037/ atm-21-1614

Data Sharing Statement: Available at http://dx.doi. org/10.21037/atm-21-1614

Conflicts of Interest: All authors have completed the ICMJE uniform disclosure form (available at http://dx.doi. org/10.21037/atm-21-1614). The authors have no conflicts of interest to declare.

Ethical Statement: The authors are accountable for all aspects of the work in ensuring that questions related to the accuracy or integrity of any part of the work are appropriately investigated and resolved. All procedures performed in this study involving human participants were in accordance with the Declaration of Helsinki (as revised in 2013). The study was approved by the Ethics Committee of The Second Affiliated Hospital of Zhengzhou University (No.: 2017663) and informed consent was taken from all the patients.
Open Access Statement: This is an Open Access article distributed in accordance with the Creative Commons Attribution-NonCommercial-NoDerivs 4.0 International License (CC BY-NC-ND 4.0), which permits the noncommercial replication and distribution of the article with the strict proviso that no changes or edits are made and the original work is properly cited (including links to both the formal publication through the relevant DOI and the license). See: https://creativecommons.org/licenses/by-nc-nd/4.0/.

\section{References}

1. Teede HJ, Misso ML, Boyle JA, et al. International PCOS Network. Translation and implementation of the Australian-led PCOS guideline: clinical summary and translation resources from the International Evidencebased Guideline for the Assessment and Management of Polycystic Ovary Syndrome. Med J Aust 2018;209:S3-8.

2. Norman RJ, Dewailly D, Legro RS, et al. Polycystic ovary syndrome. Lancet 2007;370:685-697.

3. Guzick DS. Polycystic ovary syndrome. Obstet Gynecol 2004;103:181-93.

4. Chen B, Xu P, Wang J, et al. The role of MiRNA in polycystic ovary syndrome (PCOS). Gene 2019;706:91-96.

5. Zhou JY, Zhang XY, Yu ML, et al. Effect of transcutaneuos acupoint electrostimulation on serum sex hormone levels and expression of ovarian steroid hormone metabolic 
enzymes in polycystic ovary syndrome rats. Zhen Ci Yan Jiu 2016;41:11-7.

6. Jiang YC, Ma JX. The role of MiR-324-3p in polycystic ovary syndrome (PCOS) via targeting WNT2B. Eur Rev Med Pharmacol Sci 2018;22:3286-93.

7. Sirotkin AV, Lauková M, Ovcharenko D, et al. Identification of microRNAs controlling human ovarian cell proliferation and apoptosis. J Cell Physiol 2010;223:49-56.

8. Bao Y, Chen B, Wu Q, et al. Overexpression of miR-664 is associated with enhanced osteosarcoma cell migration and invasion ability via targeting SOX7. Clin Exp Med 2017;17:51-8.

9. Wu L, Li Y, Li J, et al. MicroRNA-664 Targets Insulin Receptor Substrate 1 to Suppress Cell Proliferation and Invasion in Breast Cancer. Oncol Res 2019;27:459-67.

10. Vogler M. BCL2A1: the underdog in the BCL2 family. Cell Death Differ 2012;19:67-74.

11. Haq R, Yokoyama S, Hawryluk EB, et al. BCL2A1 is a lineage-specific antiapoptotic melanoma oncogene that confers resistance to BRAF inhibition. Proc Natl Acad Sci U S A 2013;110:4321-6.

12. Sochalska M, Schuler F, Weiss JG, et al. MYC selects against reduced BCL2A1/A1 protein expression during $\mathrm{B}$ cell lymphomagenesis. Oncogene 2017;36:2066-73.

13. Rotterdam ESHRE/ASRM-Sponsored PCOS Consensus Workshop Group. Revised 2003 consensus on diagnostic criteria and long-term health risks related to polycystic ovary syndrome. Fertil Steril 2004;81:19-25.

14. Yildiz BO, Bozdag G, Yapici Z, et al. Prevalence, phenotype and cardiometabolic risk of polycystic ovary syndrome under different diagnostic criteria. Hum Reprod 2012; 27:3067-3073.

15. Färkkilä A, Haltia UM, Tapper J, et al. Pathogenesis and treatment of adult-type granulosa cell tumor of the ovary. Ann Med 2017; 49:435-47.

16. Yildiz BO, Bozdag G, Yapici Z, et al. Prevalence, phenotype and cardiometabolic risk of polycystic ovary syndrome under different diagnostic criteria. Hum Reprod 2012;27:3067-73.

17. Liu T, Li Q, Wang S, et al. Transplantation of ovarian

Cite this article as: $\mathrm{He} \mathrm{M,} \mathrm{Mao} \mathrm{G,} \mathrm{Xiang} \mathrm{Y,} \mathrm{Li} \mathrm{P,} \mathrm{Wu} \mathrm{Y,}$ Zhao D, Li T. MicroRNA-664a-3p inhibits the proliferation of ovarian granulosa cells in polycystic ovary syndrome and promotes apoptosis by targeting BCL2A1. Ann Transl Med 2021;9(10):852. doi: 10.21037/atm-21-1614 granulosa-like cells derived from human induced pluripotent stem cells for the treatment of murine premature ovarian failure. Mol Med Rep 2016;13:5053-8.

18. Tesfaye D, Gebremedhn S, Salilew-Wondim D, et al. MicroRNAs: tiny molecules with a significant role in mammalian follicular and oocyte development. Reproduction 2018;155:R121-35.

19. Tu J, Cheung AH, Chan CL, et al. The Role of microRNAs in Ovarian Granulosa Cells in Health and Disease. Front Endocrinol (Lausanne) 2019;10:174.

20. Yoneda T, Tomofuji T, Ekuni D, et al. Serum microRNAs and chronic periodontitis: A case-control study. Arch Oral Biol 2019;101:57-63.

21. Modak JM, Roy-O'Reilly M, Zhu L, et al. Differential MicroRibonucleic Acid Expression in Cardioembolic Stroke. J Stroke Cerebrovasc Dis 2019;28:121-4.

22. Wang L, Li B, Zhang L, et al. miR-664a-3p functions as an oncogene by targeting Hippo pathway in the development of gastric cancer. Cell Prolif 2019;52:e12567.

23. Zhong S, Chen C, Liu N, et al. Overexpression Of hsamiR-664a-3p Is Associated With Cigarette SmokeInduced Chronic Obstructive Pulmonary Disease Via Targeting FHL1. Int J Chron Obstruct Pulmon Dis 2019;14:2319-29.

24. Lionnard L, Duc P, Brennan MS, et al. TRIM17 and TRIM28 antagonistically regulate the ubiquitination and anti-apoptotic activity of BCL2A1. Cell Death Differ 2019;26:902-17.

25. Haq R, Yokoyama S, Hawryluk EB, et al. BCL2A1 is a lineage-specific antiapoptotic melanoma oncogene that confers resistance to BRAF inhibition. Proc Natl Acad Sci U S A 2013;110:4321-6.

26. Wang T, Li F, Tang S. MiR-30a upregulates BCL2A1, IER3 and cyclin D2 expression by targeting FOXL2. Oncol Lett 2015;9:967-71.

27. Yu B, You W, Chen G, et al. MiR-140-5p inhibits cell proliferation and metastasis by regulating MUC1 via BCL2A1/MAPK pathway in triple negative breast cancer. Cell Cycle 2019;18:2641-50.

(English Language Editor: A. Kassem) 